\title{
Histological Transformation to Large Cell Neuroendocrine Carcinoma from Lung Adenocarcinoma Harboring an EGFR Mutation: An Autopsy Case Report
}

\author{
Rika Moriya ${ }^{1}$, Satoshi Hokari ${ }^{1}$, Satoshi Shibata ${ }^{1}$, Takeshi Koizumi ${ }^{1}$, Takafumi Tetsuka ${ }^{2}$, \\ Kazuhiko Ito ${ }^{2}$, Hideki Hashidate ${ }^{3}$ and Hiroki Tsukada ${ }^{2}$
}

\begin{abstract}
We herein report a 58-year-old Japanese woman who survived 14 years after surgery for lung adenocarcinoma harboring an epidermal growth factor receptor $(E G F R)$ exon 19 deletion. She developed recurrence, for which she underwent multimodal therapy, including EGFR-tyrosine kinase inhibitor (TKI) administration. She ultimately died from a rapidly progressive right lung tumor that was resistant to EGFR-TKI. According to the autopsy findings, she had combined large-cell neuroendocrine carcinoma (LCNEC) and adenocarcinoma in the right lung, which retained an EGFR exon 19 deletion in both components. Therefore, the histological transformation to LCNEC can be a mechanism of acquired EGFR-TKI resistance.
\end{abstract}

Key words: large-cell neuroendocrine carcinoma, adenocarcinoma, epidermal growth factor receptor tyrosine kinase inhibitor, histological transformation

(Intern Med 56: 2013-2017, 2017)

(DOI: 10.2169/internalmedicine.56.7452)

\section{Introduction}

Epidermal growth factor receptor $(E G F R)$ mutations are present in from 10-20\% of all non-small cell lung cancers (NSCLCs) and predict a response to EGFR-tyrosine kinase inhibitors (TKIs) (1). Although EGFR-TKIs are an effective treatment for these carcinomas, most patients ultimately acquire resistance (2). Recent studies have elucidated some of the mechanisms underlying EGFR-TKI resistance, including pathological transformation into small cell lung cancer (SCLC) (3). We herein report a rare case of a patient with adenocarcinoma that transformed into large-cell neuroendocrine carcinoma (LCNEC) at the time that EGFR-TKI resistance developed.

\section{Case Report}

A 58-year-old Japanese woman presented to our institution with a chest radiograph abnormality in 1999. She was a life-long non-smoker with no previous medical problems. A physical examination and laboratory data were unremarkable. A computed tomography (CT) scan revealed a $25-\mathrm{mm}$ nodule in the left $\mathrm{S} 8$ of the lung. Based on the subsequent workup, this was classified as cT1bNOM0, stage IB (TNM classification 7th edition) lung adenocarcinoma. She underwent a left lower lobe lobectomy and was diagnosed with well-differentiated lung adenocarcinoma. However, mediastinal lymph node metastases were pathologically detected. She was ultimately diagnosed with pT1bN2M0, stage IIIA (TNM classification 7th edition) lung adenocarcinoma. In the retrospective examination, the surgical specimen showed an $E G F R$ exon 19 deletion. She was administered adjuvant chemotherapy with cisplatin, mitomycin, and vindesine, and then was changed to an oral tegafur-uracil regimen because of the side effects associated with the initial regimen.

In March 2002, she developed bilateral multiple intrapulmonary metastases and was given carboplatin and paclitaxel. Gefitinib was then administered in November 2002. During a follow-up examination in 2004, brain metastases were

${ }^{1}$ Department of Respiratory Medicine and Infectious Diseases, Niigata University Graduate School of Medical and Dental Sciences, Japan, ${ }^{2}$ Department of Respiratory Medicine, Niigata City General Hospital, Japan and ${ }^{3}$ Department of Pathology, Niigata City General Hospital, Japan Received for publication March 21, 2016; Accepted for publication September 6, 2016 Correspondence to Dr. Satoshi Hokari, g1jockey@nifty.com 

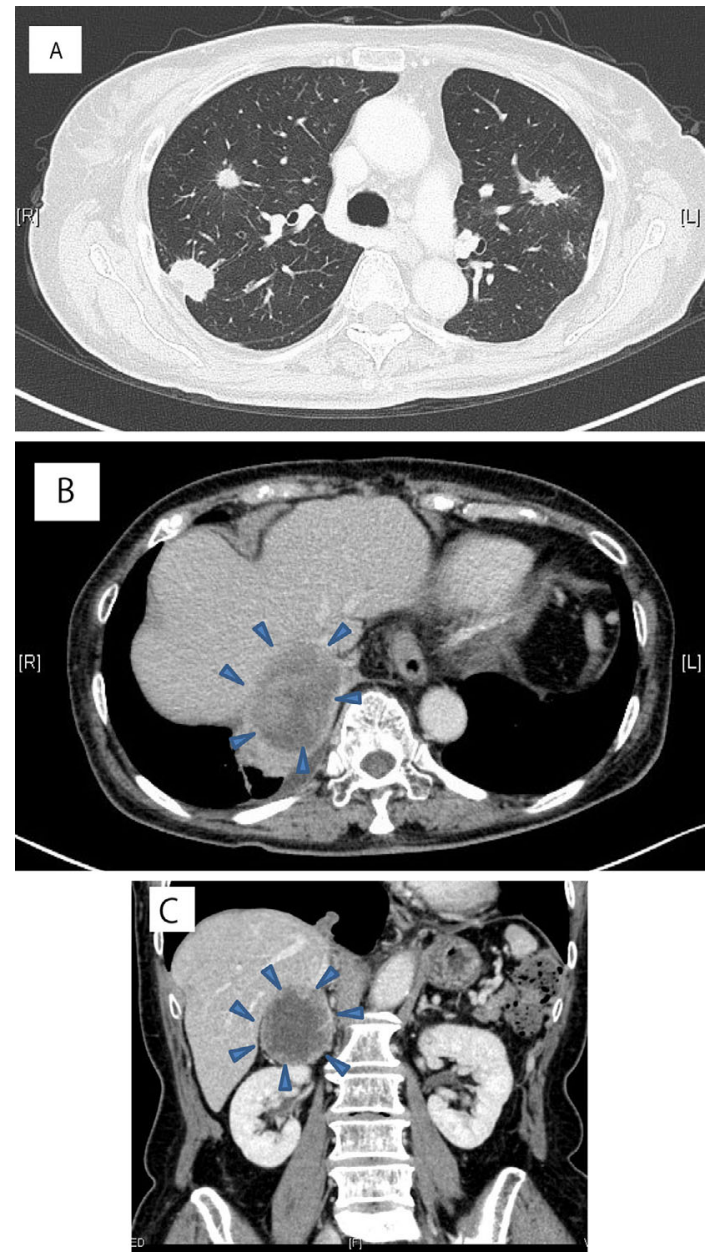

Figure 1. Computed tomography (CT) scans obtained in 2010. (A) A chest CT scan revealed intrapulmonary metastases in both lungs. (B, C) An abdominal CT scan revealed a huge right adrenal metastasis (arrowheads).

identified by magnetic resonance imaging, and the metastases were treated with brain gamma knife radiosurgery that was repeated each time new lesions emerged (seven times in total). In 2010, a CT scan revealed that her intrapulmonary metastases had progressed (Fig. 1A). Although she was administered docetaxel while receiving gefitinib treatment, a right adrenal metastasis was detected (Fig. 1B and C). She was therefore administered erlotinib in July 2010, and the disease stabilized. Our biggest concern was that the massive adrenal metastasis might rupture or cause symptoms associated with increased pressure, and we therefore performed right adrenal resection in January 2011. The immunohistochemical results revealed that it was a metastasis from the lung (Fig. 2), and a fragment analysis detected an EGFR exon 19 deletion (4).

Liver metastasis was detected in December 2011, and the patient was administered chemotherapy with pemetrexed, gefitinib, gemcitabine, and vinorelbine, but these regimens all proved to be ineffective. Notably, the metastases in the right lower lobe of lung and liver progressed rapidly in comparison to other metastases (Fig. 3). In January 2013, she was admitted to the hospital due to bacterial pneumonia and

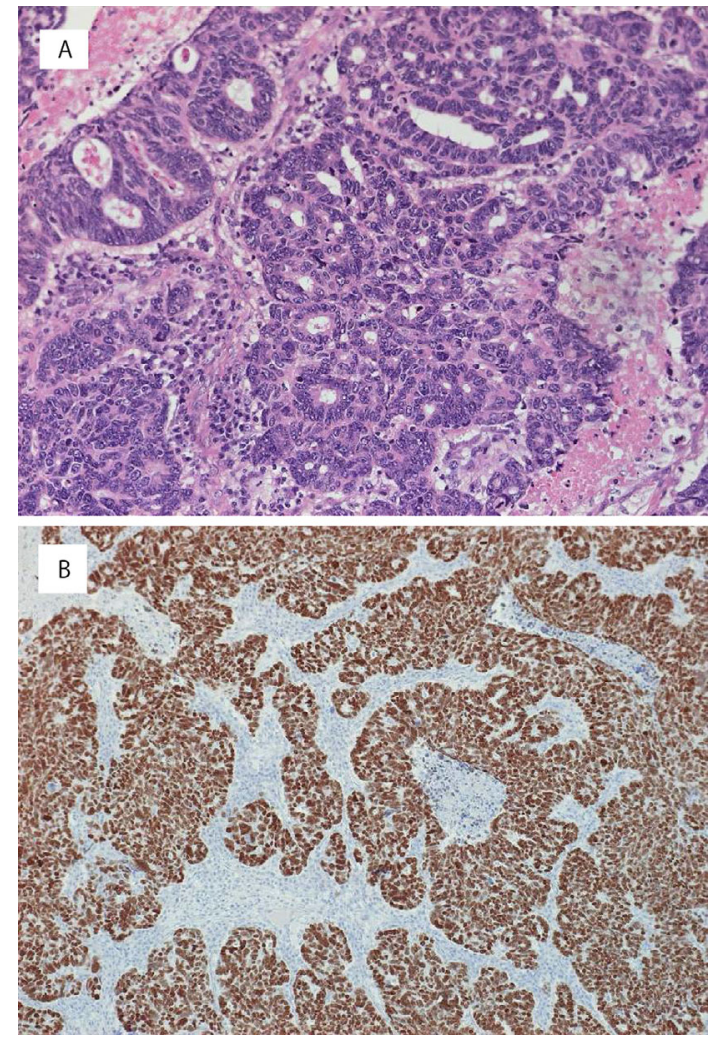

Figure 2. Microscopic findings of the resected right adrenal metastasis. (A) Hematoxylin and Eosin staining of the adrenal specimen showed that the tumor was poorly to moderately differentiated adenocarcinoma. (B) Immunohistochemical staining revealed that the specimen was positive for thyroid transcription factor-1, suggesting that these were metastases from the primary lung adenocarcinoma.
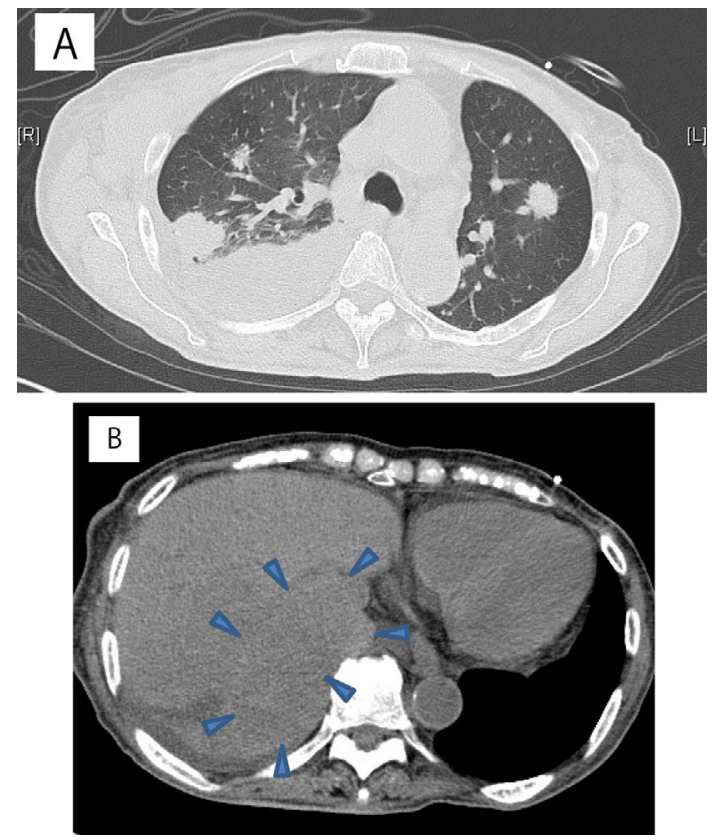

Figure 3. Computed tomography (CT) scans obtained in 2013. The largest mass in the right lower lobe of the lung (A) and the liver metastases (B) grew more rapidly than the other metastases, which was consistent with the emergence of a tumor with higher-grade morphology. 
Table 1. The Patient's History of Anticancer Treatments.

\begin{tabular}{ll}
\hline \multicolumn{1}{c}{ Date } & \multicolumn{1}{c}{ Treatment } \\
\hline September 1999 & Right upper lobe lobectomy followed by adjuvant chemotherapy \\
March 2002 & Carboplatin-paclitaxel chemotherapy \\
November 2002 & Gefitinib \\
April 2004 & Gamma knife radiosurgery for brain metastases (repeated seven times) under continuation of gefitinib \\
January 2010 & Docetaxel added on gefitinib \\
July 2010 & Erlotinib \\
January 2011 & Resection of right adrenal metastasis under continuation of erlotinib \\
January 2012 & Pemetrexed \\
May 2012 & Gefitinib re-challenge \\
August 2012 & Gemcitabine \\
December 2012 & Vinorelbine \\
\hline
\end{tabular}

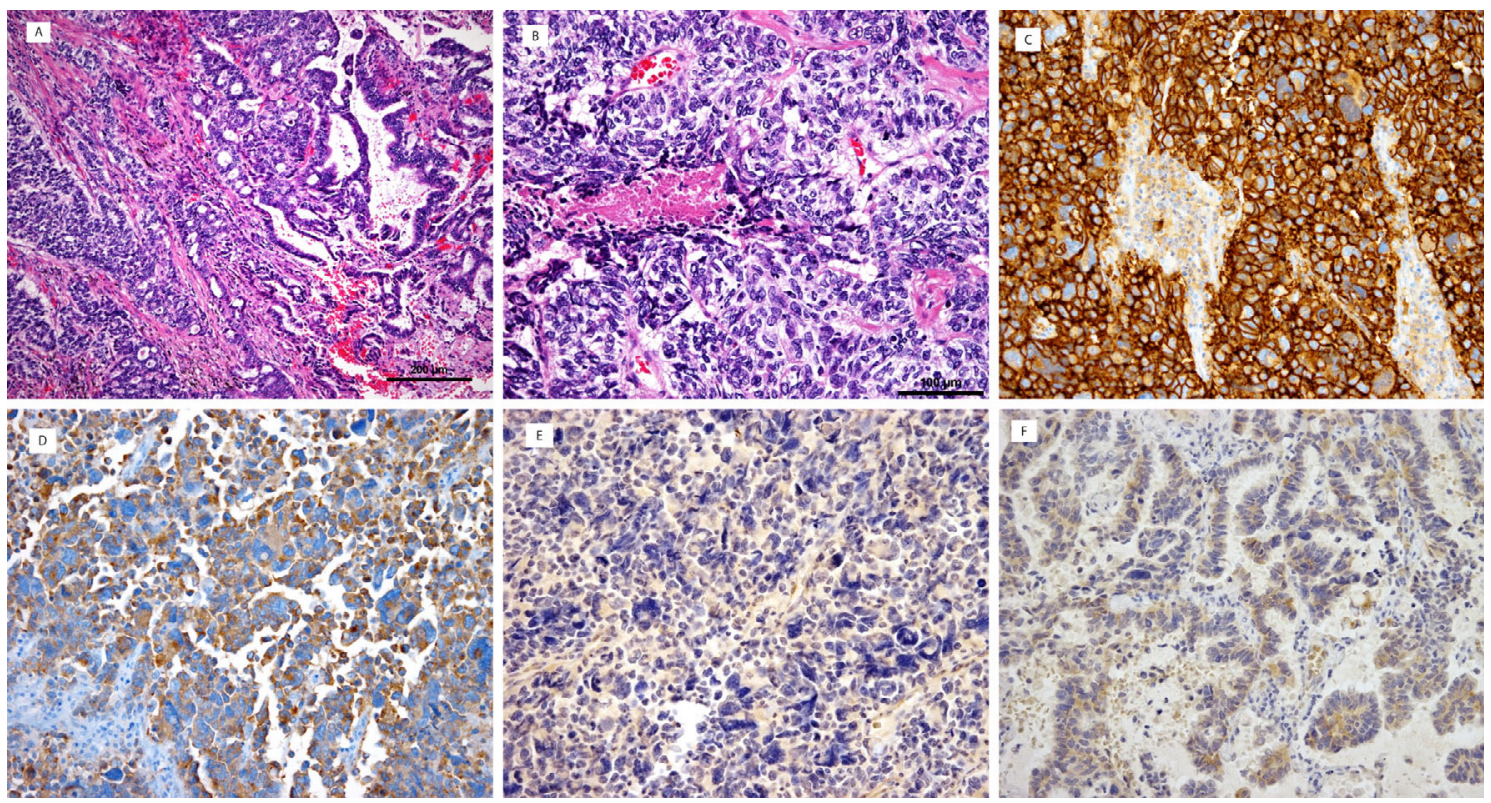

Figure 4. Autopsy specimen of the largest mass in the right lower lobe of the lung. (A) Hematoxylin and Eosin (H\&E) staining of the largest mass in the right lower lobe of the lung showed a transitional zone of well-to-moderately differentiated adenocarcinoma and neuroendocrine morphology. (B) H\&E staining of the neuroendocrine tumor portion revealed that the tumor grew in sheets and rosette-like structures and exhibited necrosis. The tumor cells were large and had abundant cytoplasm and prominent nucleoli. The neuroendocrine tumor portion was positive for neural cell adhesion molecule (C) and synaptophysin (D), supporting a diagnosis of large-cell neuroendocrine carcinoma (LCNEC). Both the LCNEC (E) and adenocarcinoma portions (F) of the lesion expressed an EGFR mutation with an exon 19 deletion.

eventually experienced fatal cardiac arrest in April of that year. The patient's history of anticancer treatments is shown in Table 1.

Autopsy findings revealed that the largest mass in the right lower lobe of the lung had directly invaded the epicardium and the liver. Using microscopy, phenotypic heterogeneity was observed among the different metastatic disease sites. Adenocarcinoma was detected in the intrapulmonary metastases, neuroendocrine tumors had disseminated through the peritoneum, and metastases were present in the liver and the epicardium. Surprisingly, these two morphologies and the transitional zone were both found in the largest lung mass (Fig. 4A). The neuroendocrine tumor grew in sheets and rosette-like structures and exhibited necrosis. The tumor cells were large and had abundant cytoplasm and prominent nucleoli (Fig. 4B). An immunohistochemical analysis revealed that the neuroendocrine component was positive for neural cell adhesion molecule (NCAM), synaptophysin, and Ki-67 (Fig. 4C and D). In contrast, the adenocarcinoma portion was negative for NCAM and synaptophysin. Although LCNEC is often difficult to distinguish from SCLC, this neuroendocrine component was consistent with LCNEC. Notably, the EGFR mutation from the antecedent adenocarcinoma was retained in both components (Fig. 4E and F). 
Table 2. Histological Diagnosis and EGFR mutation Status.

\begin{tabular}{llll}
\hline Specimen & Organ & Histological diagnosis & EGFR mutaion \\
\hline Surgery in 1999 & Lung (left lower lobe; primary tumor) & Adenocarcinoma & exon 19 del., T790M (-)* \\
& Mediastinum lymph node & Adenocarcinoma & exon 19 del., T790M (-)* \\
\hline Surgery in 2011 & Right adrenal gland & Adenocarcinoma & exon 19 del. \\
\hline Autopsy in 2013 & Lung (multiple intrapulmonary metastases) & Adenocarcinoma & exon 19 del., T790M (-)* \\
& Mediastinum lymph nodes & Adenocarcinoma & exon 19 del. \\
& Pleural dissemination (left) & Adenocarcinoma & exon 19 del. (both components) \\
& Lung (right lower lobe; metastatic tumor) & Combined LCNEC and adenocarcinoma 19 del. & exon 19 del. \\
& Pleural dissemination (right) & LCNEC & LCNEC \\
& Pericardium (invasive lesion) & LCNEC & LCNEC \\
& Liver (right lobe) & LCNEC & exon 19 del., T790M (-)* \\
& Paritoneum dissemination & exon 19 del. &
\end{tabular}

EGFR: epidermal growth factor receptor, exon 19 del.: exon 19 deletion, LCNEC: large-cell neuroendocrine carcinoma, N/E: not examined

*EGFR T790M mutation was examined using the Scorpion amplification refractory mutation system method.

The histological diagnosis and EGFR mutation status are summarized in Table 2.

\section{Discussion}

We herein report a case of lung adenocarcinoma that transformed to LCNEC and became resistant to EGFR-TKIs. LCNEC of the lung is a subtype of large-cell carcinoma and generally has a poor prognosis compared to other NSCLCs (5). Although a case of combined LCNEC and adenocarcinoma with an EGFR mutation has been reported (6), it is unlikely that the present patient originally had a combined LCNEC because a transitional zone between the two components was detected in the autopsy findings, despite no LCNEC portion existing in the lobectomy specimen. Furthermore, it is unlikely that a patient with recurrent LCNEC would have survived without optimal treatment for 10 years.

The development of EGFR-TKI resistance is known to be concordant with a phenotypic switch to SCLC from NSCLC (7-9). Because LCNEC is very similar to SCLC (10), we believe that histological transformation to LCNEC can be a mechanism of acquired EGFR-TKI resistance. Recently, Kogo et al. reported a case of EGFR mutant adenocarcinoma that transformed to LCNEC, which was diagnosed by bronchoscopic tumor resection (11). In their case, the patient had received long-term treatment for lung adenocarcinoma with many chemotherapy regimens including EGFR-TKIs, as performed in the present case. A single tumor that expresses an EGFR mutation in cells of many different morphologies may reflect the existence of cancer stem cells. In our case, we suspect that either minor clones with stem cell-like properties were selected for when the major clones were eliminated by EGFR-TKI treatment or that the EGFR-TKI treatment induced stem cell-like properties within cells (12).

Besides histological transformation, several mechanisms of acquired resistance have been reported, such as secondary
EGFR mutations and the activation of collateral EGFR signaling pathways (3). With respect to secondary mutations, EGFR T790M mutation is well-known and develops in 50\% of lung adenocarcinomas that acquire resistance to EGFRTKIs. Recently, inter-tumor heterogeneity was reported as a mechanism of acquired resistance to EGFR-TKIs. Furugen et al. reported a case in which the patient developed EGFRTKI resistance via SCLC transformation and an EGFR T790M mutation in separate metastatic organs (13). In addition, several reports have suggested a reciprocal relationship between a T790M mutation and histologic transformation $(14,15)$. Although an EGFR T790M mutation was not detected in our case, it is possible that another resistance mechanism was present.

This is a rare case report of transformed LCNEC after EGFR-TKI exposure, although histological transformation in this setting is not uncommon. Sequist et al. reported that $14 \%$ of patients who underwent tumor biopsies experienced a fundamental histological transformation into SCLC after acquiring EGFR-TKI resistance (3). In the present case, we emphasize the value of repeatedly assessing cancers throughout the course of the disease and the importance of extending our understanding of drug resistance, which in turn would allow for the determination of the best treatment option for each patient. However, our case involved multiple organ metastases, making it difficult to select an appropriate biopsy site. Yanagisawa et al. reported a case of a patient with lung adenocarcinoma that expressed an EGFR mutation who had a lung mass that gradually enlarged despite treatment with erlotinib, although the other metastatic lesions remained the same size. The patient underwent a second lung biopsy via video-assisted thoracoscopic surgery and was ultimately diagnosed with LCNEC, which might have resulted from transformation of adenocarcinoma, as with our case (16). When EGFR-TKI resistance is detected, a second histopathological examination should be considered, especially with a rapidly progressing or highly invasive lesion.

Although cases of small cell transformation have been 
previously reported to be responsive to regimens used to treat $\operatorname{SCLC}(3,8)$, there are few reports about the treatment of LCNEC transformed from adenocarcinoma. However, LCNEC responds well to SCLC regimens, compared with traditional NSCLC treatments (17). Additionally, Kogo et al. reported a case of LCNEC transformation in which the patient was treated with SCLC regimens and obtained a partial response (11). We believe the present LCNEC could have been treated with effective regimens, such as amrubicin or irinotecan, if the patient had been diagnosed with LCNEC earlier.

In summary, we herein reported an autopsy case of LCNEC that transformed from adenocarcinoma harboring an $E G F R$ mutation. The findings from this case showed the clinical importance of pathological assessment at the time of progression in patients with tumors that express an EGFR mutation. Further investigation is necessary to clarify the EGFR-TKI resistance mechanisms involved in transformation to LCNEC.

The authors state that they have no Conflict of Interest (COI).

\section{References}

1. Lynch TJ, Bell DW, Sordella R, et al. Activating mutations in the epidermal growth factor receptor underlying responsiveness of non-small cell lung cancer to gefitinib. N Engl J Med 350: 21292139, 2004.

2. Jackman D, Pao W, Riely GJ, et al. Clinical definition of acquired resistance to epidermal growth factor receptor tyrosine kinase inhibitors in non-small-cell lung cancer. J Clin Oncol 28: 357-360, 2010 .

3. Sequist LV, Waltman BA, Dias-Santagata D, et al. Genotypic and histological evolution of lung cancers acquiring resistance to EGFR inhibitors. Sci Transl Med 3: 75ra26, 2011.

4. Yatabe Y, Hida T, Horio Y, Kosaka T, Takahashi T, Mitsudomi T. A rapid, sensitive assay to detect EGFR mutation in small biopsy specimens from lung cancer. J Mol Diagn 8: 335-341, 2006.

5. Fasano M, Della Corte CM, Papaccio F, Ciardiello F, Morgillo F. Pulmonary large-cell neuroendocrine carcinoma: form epidemiology to therapy. J Thorac Oncol 10: 1133-1141, 2015.

6. Yoshida Y, Ota S, Murakawa T, Takai D, Nakajima J. Combined large cell neuroendocrine carcinoma and adenocarcinoma with epidermal growth factor receptor mutation in a female patient who never smoked. Ann Thorac Cardiovasc Surg 20 Suppl: 582-584,
2014.

7. Arcila ME, Oxnard GR, Nafa K, et al. Rebiopsy of lung cancer patients with acquired resistance to EGFR inhibitors and enhanced detection of the T790M mutation using a locked nucleic acidbased assay. Clin Cancer Res 17: 1169-1180, 2011.

8. Morinaga R, Okamoto I, Furuta K, et al. Sequential occurrence of non-small cell and small cell lung cancer with the same EGFR mutation. Lung Cancer 58: 411-413, 2007.

9. Zhang Y, Li XY, Tang Y, et al. Rapid increase of serum neuron specific enolase level and tachyphylaxis of EGFR-tyrosine kinase inhibitor indicate small cell lung cancer transformation from EGFR positive lung adenocarcinoma? Lung Cancer 81: 302-305, 2013.

10. Rossi G, Cavazza A, Marchioni A, et al. Role of chemotherapy and the receptor tyrosine kinase KIT, PDGFR $\alpha, \operatorname{PDGFR} \beta$, and Met in large-cell neuroendocrine carcinoma of the lung. J Clin Oncol 23: 8774-8785, 2005.

11. Kogo M, Shimizu R, Uehara K, et al. Transformation to large cell neuroendocrine carcinoma as acquired resistance mechanism of EGFR tyrosine kinase inhibitor. Lung Cancer 90: 364-368, 2015.

12. Shien $K$, Toyooka $S$, Yamamoto $H$, et al. Acquired resistance to EGFR inhibitors is associated with a manifestation of stem celllike properties in cancer cells. Cancer Res 73: 3051-3061, 2013.

13. Furugen M, Uechi K, Hirai J, et al. An autopsy case of two distinct, acquired drug resistance mechanisms in epidermal growth factor receptor-mutant lung adenocarcinoma: small cell carcinoma transformation and epidermal growth factor receptor T790M mutation. Intern Med 54: 2491-2496, 2015.

14. Scher KS, Saldivar JS, Fishbein M, Marchevsky A, Reckamp KL. EGFR-mutated lung cancer with $T 790 M$-acquired resistance in the brain and histologic transformation in the lung. $\mathrm{J}$ Natl Compr Canc Netw 11: 1040-1044, 2013.

15. Suda K, Murakami I, Sakai K, et al. Small cell lung cancer transformation and T790M mutation: complimentary roles in acquired resistance to kinase inhibitors in lung cancer. Sci Rep 5: 14447, 2015.

16. Yanagisawa S, Morikawa N, Kimura Y, Nagano Y, Murakami K, Tabata T. Large-cell neuroendocrine carcinoma with epidermal growth factor receptor mutation: possible transformation of lung adenocarcinoma. Respirology 17: 1275-1277, 2012.

17. Igawa $\mathrm{S}$, Watanabe $\mathrm{R}$, Ito $\mathrm{H}$, et al. Comparison of chemotherapy for unresectable pulmonary high-grade non-small cell neuroendocrine carcinoma and small-cell lung cancer. Lung Cancer 68: 438445, 2010.

The Internal Medicine is an Open Access article distributed under the Creative Commons Attribution-NonCommercial-NoDerivatives 4.0 International License. To view the details of this license, please visit (https://creativecommons.org/licenses/ by-nc-nd/4.0/).

(C) 2017 The Japanese Society of Internal Medicine http://www.naika.or.jp/imonline/index.html 